\title{
Novel MMC control for active balancing and minimum ripple current in series-connected battery strings
}

\author{
Damien F. Frost \\ University of Oxford \\ 17 Parks Road \\ Oxford, United Kingdom \\ Phone: +44 (0) 1865-273004 \\ Email: damien.frost@eng.ox.ac.uk \\ URL: epg.eng.ox.ac.uk
}

\author{
Damien A. Howey \\ University of Oxford \\ 17 Parks Road \\ Oxford, United Kingdom \\ Phone: +44 (0) 1865-273004 \\ Email: david.howey@eng.ox.ac.uk \\ URL: epg.eng.ox.ac.uk
}

\begin{abstract}
The performance of a string of series-connected batteries is typically restricted by the worst battery cell in the string and a single failure point will render the entire string unusable. To address these issues, we present a decentralised battery management system based on a modular multilevel converter (MMC) with a distributed inductor. This novel MMC design enables each MMC cell to operate autonomously, without central control, by sensing the local inductor voltage. Batteries are loaded with a current proportional to their capacity, and the MMC cells also work together to minimize the output ripple voltage of the full series stack. We show experimentally that the novel decentralized controller implemented in each MMC cell can reduce the output voltage ripple by $69 \%$. We also give an extensive theoretical analysis of the waveforms produced by the MMC using Fourier decomposition. This analysis has applications in all modular multilevel converters.
\end{abstract}

\section{INTRODUCTION}

Power electronics is playing a key role in the reduction of greenhouse gas emissions through the integration of green energy technologies with the grid [1],[2]. One area of increased power electronics penetration is electrical energy storage systems such as lithium-ion battery packs [3], where the market is growing very rapidly [4]. Rapid adoption of grid-connected energy storage requires state of the art power electronic converters and energy management systems. Furthermore, since the energy storage device (batteries, for example) is the most expensive component [5], the power electronics should be designed to maximise performance and lifetime whilst ensuring safety [6], [7]. In the worst case scenario, an unmanaged series string of batteries will be limited by the weakest cell [8], thus a single failed cell can render the entire string useless.

In energy storage systems that use electrochemical cells, long term pack performance can be greatly improved by active energy management at the individual cell level, by placing an individual power converter and battery management system on every cell [9], [10]. Recent advancements in wide band gap switching devices, whose cost is expected to decrease over time, will increase conversion efficiencies (and reduce power losses), and reduce overall system costs through higher power density [11], [12], [13] and decreased cooling requirements [14].

For certain cell chemistries, individual cell monitoring is necessary, and active cell balancing enhances performance. As an example, lithium ion battery technology is unsafe if overcharged, where cells may explode or rupture [15], [16]. Therefore, in order to maximize the energy storage potential of several cells connected in series and in parallel, the state of charge of individual cells in a battery pack must be measured and balanced.

We present work on a cell level battery management system and power converter which uses a decentralized controller strategy to regulate the state of charge of serially connected cells of varying capacities. The distributed battery management system is designed to discharge and charge each cell in a series string of cells proportional to its capacity. The converters are controlled in such a way as to minimize the size of the filtering components in the series string.

\section{System ARChiteCTURE}

An example of the proposed system architecture is shown in Fig. 1. In this example, the system is constructed out of three MMC cells each containing a half bridge switching network similar to the switching networks found in the sub-modules of MMC converters [17]. However each submodule, which we shall in this paper call a 'smart cell', has two distinct features that make it different from a standard MMC submodule:

1) There exists an independent, autonomous controller in each smart cell.

2) Each smart cell includes a small filter inductor, $L_{s c}$.

The autonomous controllers are used to synchronize the switching events of all of the sub-modules in a string of smart cells to minimize the overall ripple voltage at the output, $v_{\text {out }}$. Each autonomous controller also manages the state of charge (SOC), and monitors the state of health $(\mathrm{SOH})$ of its 


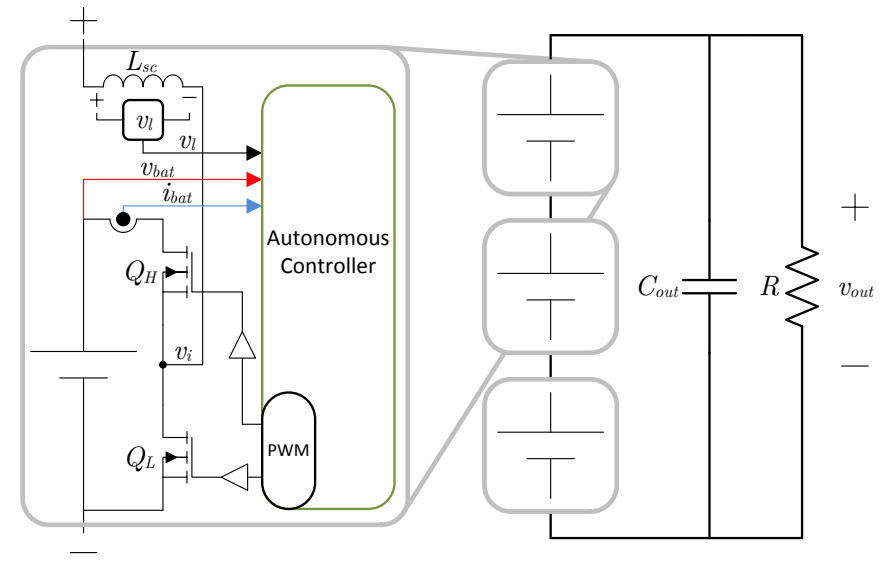

Figure 1: An example of a system containing three 'smart cells'.

locally connected battery cell. This information is used to apply a duty cycle to the switches $Q_{L}$ and $Q_{H}$, such that the connected battery cell discharges in proportion to its capacity. Discharging all battery cells in proportion to their respective capacities yields two large benefits for the string of smart cells:

1) The SOC of all of the battery cells in the string will be synchronized.

2) Larger, healthier battery cells will be loaded more than the smaller, more degraded battery cells, thus the pack will degrade at a more uniform rate [18].

The voltage across the small filter inductor $L_{S c}$ is used by the autonomous local controller to determine the optimal switch timings for $Q_{H}$ and $Q_{L}$. Furthermore, by splitting the output filter inductor amongst every smart cell, the inductance is reduced. Eventually $L_{s c}$ could be small enough to be implemented on the trace of a PCB, greatly reducing the cost and size of this component. The dc output load simply requires a filter capacitor, $C_{\text {out }}$.

\section{Optimal Switching PATtern}

In order to minimize the output voltage ripple measured at $v_{\text {out }}$ an optimal switching pattern of all of the switches $Q_{H}$ and $Q_{L}$ must be determined. This is done by all smart cells collectively minimizing the ac rms inductor current. To help solve this problem, a few assumptions will be made about the system of smart cells.

\section{A. Problem and Assumptions}

Given set of $M$ battery cells $B_{i}=\left\{B_{1}, B_{2}, \ldots, B_{M}\right\}$ with capacities $C_{i}=\left\{C_{1}, C_{2}, \ldots, C_{M}\right\}$, our objective is to find a set of phases, $\phi_{i}=\left\{\phi_{1}, \phi_{2}, \ldots, \phi_{M}\right\}$ for the turn-on of each smart cell which will minimize the ripple current in local inductor $L_{s c}$, thus minimize the output ripple voltage.

For simplicity, the nominal voltages of all of the battery cells will be $V_{\text {nom }}=V_{1}=V_{2}=\ldots=V_{M}$, which is a reasonable assumption because the SOC of all of the battery cells will be synchronized. The duty cycle of each smart cell can be calculated using the following equation:

$$
D_{i}=\frac{C_{i}}{C_{M A X}}
$$

where $C_{M A X}$ is the maximum capacity expected amongst all of the battery cells, or ideally, the maximum value found in the capacity array such that $0 \leq D_{i} \leq 1$ for all $i$.

In a series string of smart cells, the voltage applied to each inductor, $L_{s c}$, will be identical. Thus, we can group all of the distributed inductors together into a single inductor, $L=M L_{s c}$. The voltage across $L$ will be determined by the sum of all of the $v_{i}$ voltages of the smart cells, and the output dc voltage. The output dc voltage is given by (2):

$$
V_{\text {out }}=\sum_{i=1}^{M} D_{i} V_{\text {nom }}=\sum_{i=1}^{M} \frac{C_{i}}{C_{M A X}} V_{\text {nom }}=\frac{V_{\text {nom }}}{C_{M A X}} \sum_{i=1}^{M} C_{i}
$$

The ac root mean squared (rms) value of the inductor ripple current can be expressed by (3):

$$
I_{\text {Lac }-r m s}=\sqrt{\frac{1}{T_{s}} \int_{0}^{T_{s}}\left[I(s)+\frac{1}{L} \int_{s}^{t}\left(v_{\text {out }}(\tau)-V_{\text {out }}\right) d \tau\right]^{2} d t}
$$

where $I(s)$ is the anti-derivative of $\left(v_{\text {out }}(s)-V_{\text {out }}\right) / L, \tau$ and $s$ are dummy variables of integration, and $t$ is time. The variable $v_{\text {out }}(t)$ is defined by (4):

$$
v_{\text {out }}(t)=\sum_{i=1}^{M} v_{i}(t)
$$

where $v_{i}(t)$ is the output voltage of the $i^{t h}$ smart cell as measured from the negative terminal of the battery cell to the output terminal of the transistors, as shown in Fig. 1. Therefore, $v_{\text {out }}(t)$ is the output voltage of a series string of smart cells.

\section{B. Determine $I_{\text {Lac-rms }}$}

In order to determine an optimal set of phase angles $\phi_{i}$ a function which describes the output of a smart cell in terms of $\phi_{i}$ needs to be derived. The output voltage of a smart cell will generally look like a periodic rectangular wave, as shown in Fig. 2.

Using the generalized values in Fig. 2, the Fourier Series representation of the output voltage of any smart cell can be derived, and it is shown in (5) - (9).

$$
\begin{aligned}
v_{i}(t) & =d_{i}(t) V_{\text {nom }} \\
d_{i}(t) & =\frac{a_{0 i}}{2}+\sum_{n=1}^{\infty}\left[a_{n i} \cos \left(\frac{2 \pi n t}{T_{s}}\right)+b_{n i} \sin \left(\frac{2 \pi n t}{T_{s}}\right)\right](6)
\end{aligned}
$$




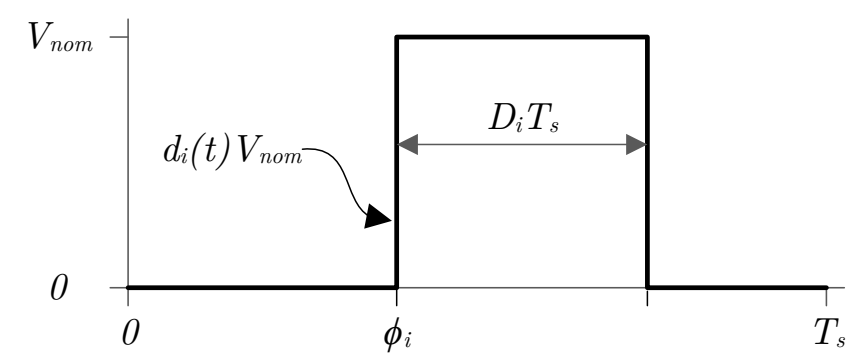

Figure 2: Output voltage, $d_{i}(t) V_{\text {nom }}$, of a smart cell with phase $\phi_{i}$, duty cycle $D_{i}$, and switching period, $T_{S}$.

$$
\begin{aligned}
& a_{0 i}=2 d_{i} \\
& a_{n i}=\frac{1}{n \pi}\left(\sin \left(2 \pi n\left(D_{i}+\frac{\phi_{i}}{T_{s}}\right)\right)-\sin \left(2 \pi n \frac{\phi_{i}}{T_{s}}\right)\right) \\
& b_{n i}=\frac{1}{n \pi}\left(\cos \left(2 \pi n \frac{\phi_{i}}{T_{s}}\right)-\cos \left(2 \pi n\left(D_{i}+\frac{\phi_{i}}{T_{s}}\right)\right)\right)
\end{aligned}
$$

Now we can substitute (5) into (4) and solve for $I_{\text {Lac-rms }}^{2}$ using (3). It can be shown that the result is:

$$
\begin{aligned}
& I_{\text {Lac-rms }}^{2}=\frac{1}{2}\left(\frac{V_{\text {nom }} T_{s}}{2 \pi L}\right)^{2} \sum_{n=1}^{\infty} \sum_{i=1}^{M} \sum_{j=1}^{M} \frac{4}{\pi^{2} n^{4}} \\
& {\left[\sin \left(\pi n D_{i}\right) \sin \left(\pi n D_{j}\right) \cos \left(\pi n\left(D_{i}-D_{j}+2 \frac{\phi_{i}}{T_{s}}-2 \frac{\phi_{j}}{T_{s}}\right)\right)\right]}
\end{aligned}
$$

Now let $\theta_{x}=2 \pi \phi_{x} / T_{s}$, so that the switching angles can be measured in radians instead of time:

$$
\begin{aligned}
I_{\text {Lac-rms }}^{2}= & \frac{1}{2}\left(\frac{V_{\text {nom }} T_{s}}{2 \pi L}\right)^{2} \sum_{n=1}^{\infty} \sum_{i=1}^{M} \sum_{j=1}^{M} \frac{4}{\pi^{2} n^{4}}\left[\sin \left(\pi n D_{i}\right)\right. \\
& \left.\cdot \sin \left(\pi n D_{j}\right) \cos \left(\pi n\left(D_{i}-D_{j}\right)+n\left(\theta_{i}-\theta_{j}\right)\right)\right]
\end{aligned}
$$

Equation (11) can now be minimized to determine an optimal set $\theta_{i}$ that will minimize the ac rms current in the inductor $L_{s c}$, and therefore, the output voltage ripple in $v_{\text {out }}$.

\section{Simplifying THE PROBLEM}

Examining (11), we immediately see that solving for an optimal set $\theta_{i}$ to minimize $I_{\text {Lac-rms }}$ is non trivial, and difficult to achieve without significant computational power and global information about the system. Therefore in this section we present a way to identify a set $\theta_{i}$ which will yield a satisfactory solution, with significantly less computational requirements, in a decentralized fashion.

First, let us represent the switching action of the $i^{\text {th }}$ smart cell as a vector, $v_{i}$, in the unit circle as depicted in Fig. 3. The $i^{t h}$ smart cell will turn on at $\theta_{i}$, and turn off at $\theta_{i}+2 \pi D_{i}$. Now define a new vector, $v_{i}^{\prime}$, whose phase places the vector half way between the turn on and turn off times and whose length is $\sin \pi D_{i}$. Therefore the phase and length of $v_{i}^{\prime}$ are given by:

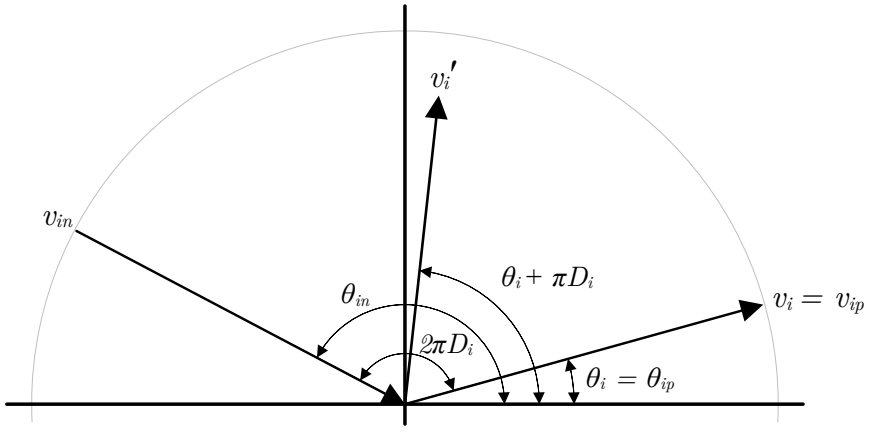

Figure 3: Representing the switching action of the $i^{\text {th }}$ smart cell as a vector, with its weighted, phase shifted vector, $v_{i}^{\prime}$. The switching actions of the $i^{t h}$ smart cell are also shown as vectors $v_{i p}$ and $v_{i n}$.

$$
\begin{aligned}
\angle v_{i}^{\prime} & =\theta_{i}^{\prime}=\theta_{i}+\pi D_{i} \\
\left|v_{i}^{\prime}\right| & =\sin \left(\pi D_{i}\right)
\end{aligned}
$$

Now apply the transformation described in (12) and (13) to all of the smart cells, and then sum all $v_{i}^{\prime}$, we can find the square of the magnitude of the total sum vector, $\left|v_{\Sigma}^{\prime}\right|^{2}$ :

$$
\left|v_{\Sigma}^{\prime}\right|^{2}=\sum_{i=1}^{M} \sum_{j=1}^{M} \sin \left(\pi D_{i}\right) \sin \left(\pi D_{j}\right) \cos \left(\pi\left(D_{i}-D_{j}\right)+\theta_{i}-\theta_{j}\right)
$$

Equation (14) has the exact same form as (11) when $n=1$ (i.e. only the fundamental is considered). Therefore, one control algorithm that will yield a good but sub-optimal minimum of (11) is to minimize the magnitude of the total sum vector, $\left|v_{\Sigma}^{\prime}\right|^{2}$. This algorithm is used in the simulation study and experimental setup, as presented in the following sections.

\section{IMPLEMENTATION OF THE CONTROL ALGORITHM}

Calculating the magnitude of the total sum vector using the formula in Equation (14) still requires accurate knowledge of the duty cycles and phase shifts of all of the other smart cells. In order to eliminate communication between smart cells, this approach is still not ideal. This section will address this issue.

\section{A. Smart cell measurements}

First, let us consider the measurements a smart cell will be able to make. By measuring the voltage across its local inductor, each smart cell will only know when other smart cells are switching on or off. These switching actions are shown in Fig. 3 as vectors $v_{i p}$ as $v_{i n}$.

Using Fig. 3 as reference, from the point of view of a single smart cell, it will see the switching actions of the $i^{\text {th }}$ smart cell as two corresponding voltage transitions: a positive voltage transition across the inductor $L_{s c}$, depicted by the vector $v_{i p}$ with switching angle $\theta_{i p}$, and its negative corresponding 
voltage transition, depicted by the vector $v_{i n}$ with switching angle $\theta_{i n}$. For a smart cell detecting only one other smart cell, the problem is very trivial and it is easy to determine not only when the other smart cell is switching, but its duty cycle as well.

The challenge arises when multiple smart cells start switching. As soon as a single smart cell needs to determine the switching angles and duty cycles of two or more other smart cells, it is impossible to match every "on" transition with its corresponding "off" transition. We will now show that this is not necessary to yield the optimal solution as defined by (14).

First, use (15) and (16) to replace all of the $D_{x} \mathrm{~s}$ and $\theta_{x} \mathrm{~s}$ with the notation of Fig. 3.

$$
\begin{aligned}
\theta_{i} & =\theta_{i p} \\
\pi D_{i} & =\left(\frac{\theta_{i n}-\theta_{i p}}{2}\right)
\end{aligned}
$$

this yields an expression in terms of measured quantities:

$$
\begin{aligned}
\left|v_{\Sigma}^{\prime}\right|^{2}=\sum_{i=1}^{M} \sum_{j=1}^{M} \sin \left(\frac{\theta_{i n}-\theta_{i p}}{2}\right) \sin \left(\frac{\theta_{j n}-\theta_{j p}}{2}\right) \\
\cdot \cos \left(\left(\frac{\theta_{i n}+\theta_{i p}}{2}\right)-\left(\frac{\theta_{j n}+\theta_{j p}}{2}\right)\right)
\end{aligned}
$$

Equation (17) gives the magnitude of the total vector in terms of quantities measured by each smart cell. However, this equation still seems to imply that the "on" transitions must be correctly paired with each of their corresponding "off" transitions. We will now show that this is not the case by first considering the case where only one set of "on" transitions are swapped. Therefore, consider a set of $M$ smart cells, where the $k^{\text {th }}$ and $l^{\text {th }}$ positive transitions are paired with the $l^{\text {th }}$ and $k^{\text {th }}$ negative transitions, respectively. It can be shown that:

$$
\begin{gathered}
\left|v_{\Sigma}^{\prime}\right|^{2}=\sum_{\substack{i=1 \\
i \neq k, i \neq l}}^{M} \sum_{\substack{j \neq 1 \\
j \neq k, j \neq l}}^{M}\left[\sin \left(\frac{\theta_{i n}-\theta_{i p}}{2}\right) \sin \left(\frac{\theta_{j n}-\theta_{j p}}{2}\right)\right. \\
\left.\cdot \cos \left(\left(\frac{\theta_{i n}+\theta_{i p}}{2}\right)-\left(\frac{\theta_{j n}+\theta_{j p}}{2}\right)\right)\right]+\sin ^{2}\left(\frac{\theta_{k n}-\theta_{l p}}{2}\right) \\
+\sin \left(\frac{\theta_{l n}-\theta_{k p}}{2}\right) \sin \left(\frac{\theta_{k n}-\theta_{l p}}{2}\right) \cos \left(\left(\frac{\theta_{l n}+\theta_{k p}}{2}\right)\right. \\
\left.-\left(\frac{\theta_{k n}+\theta_{l p}}{2}\right)\right)+\sin ^{2}\left(\frac{\theta_{l n}-\theta_{k p}}{2}\right)+\sin \left(\frac{\theta_{l n}-\theta_{k p}}{2}\right) \\
\cdot \sin \left(\frac{\theta_{l n}-\theta_{k p}}{2}\right) \cos \left(\left(\frac{\theta_{k n}+\theta_{l p}}{2}\right)-\left(\frac{\theta_{l n}+\theta_{k p}}{2}\right)\right)= \\
\sum_{i=1}^{M} \sum_{j=1}^{M} \sin \left(\frac{\theta_{i n}-\theta_{i p}}{2}\right) \sin \left(\frac{\theta_{j n}-\theta_{j p}}{2}\right) \cos \left(\left(\frac{\theta_{i n}+\theta_{i p}}{2}\right)\right. \\
\left.-\left(\frac{\theta_{j n}+\theta_{j p}}{2}\right)\right)
\end{gathered}
$$

By extension, we can swap any pair and any number of transitions with each other and get the same result. Therefore, in order to minimize the total sum vector of (14), each smart cell need only know when the positive and negative transitions occur to determine its local optimal switching pattern. Moreover, it can be shown that these results are also true for all harmonics, 1 to $n$.

\section{B. Controller Design}

A MATLAB-Simulink model of a smart cell using the theory developed in this work was built using the SimPowerSystems toolbox. The model uses the Simscape battery model and MOSFETs to simulate the power circuit. The controller was implemented as an embedded MATLAB function, and is executed once per switching cycle when the upper MOSFET, $Q_{H}$, is switched on.

Figure 4 illustrates the algorithm the controller implements as a block diagram. Steps 1 through 8 outlined in green are the steps implemented by the embedded MATLAB function.

1) Edge Detector: Detects all of the positive and negative transitions that occur during every switching cycle, $T_{s}$. The edge detector handles cases where multiple smart cells turn on or off at the same time by tracking the different voltage levels measured with $v_{l}$.

2) Transformation: The positive and negative edges are paired together to form a set of sensed smart cells. The transformation described by (12) and (13) is applied.

3) Sum all vectors: All of the vectors found and transformed are added together as described by (14)

4) Subtract local $v_{0}^{\prime}$ : The vector which describes the switching action of the local cell is subtracted from the total sum vector to yield $v_{\text {other }}^{\prime}$.

5) Calculate reference: In order to minimize the sum of the total vector, $\angle v_{\text {ref }}^{\prime}$ is calculated by simply adding $\pi$ to $\angle v_{\text {other }}^{\prime}$. In this way, the total sum vector is minimized.

6) Calculate angle: The actual phase shift of the local smart cell is calculated by reversing the transform of (12).

7) Apply limits: $\pm 2 \pi$ is added to $\angle v_{\text {ref }}$ until it lies between $-\pi$ and $\pi$.

8) Calculate phase: Finally, the phase that will be sent to the PWM generator is calculated by multiplying the phase error $\left(0-\angle v_{r e f}\right)$ by the duty cycle of the local smart cell, $D_{0}$, and a gain of $G_{P h C t r l}$. By multiplying the error by the duty cycle, the phase shifts of smart cells with different capacities will change at different rates, allowing the system to find an equilibrium point.

9) PWM Generator: $\theta_{0}^{\text {new }}$ is used by the PWM generator to produce gating signals for the MOSFETs.

\section{Simulation Study}

A simulation study of a series string of three smart cells was undertaken. Fig. 1 shows a schematic of the 3 smart cell pack. Table I lists the simulation parameters used.

The simulation consists of the three smart cells operating completely independently of each other. The optimal switching controller is turned on $1.0 \mathrm{~ms}$ into the simulation. A value of $C_{M A X}=4.00 \mathrm{Ah}$ was pre-programmed into each smart cell. 


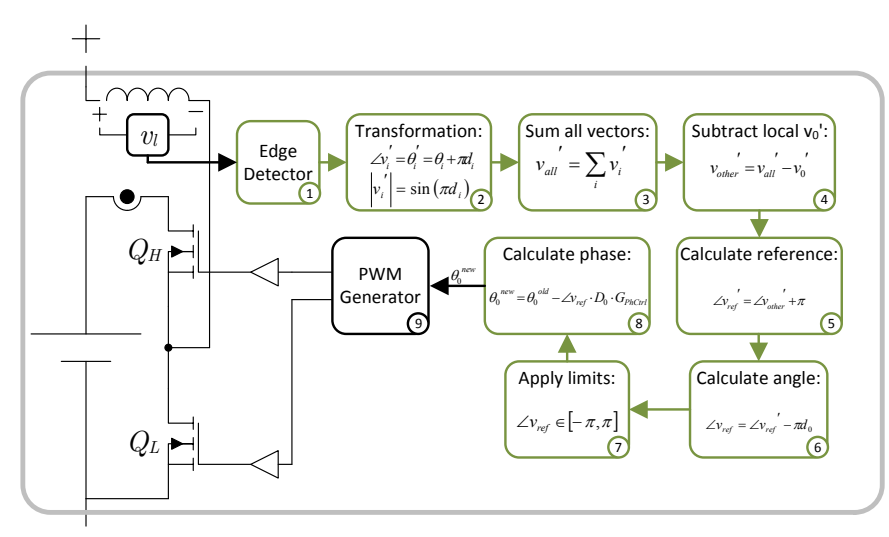

Figure 4: Control block diagram of a smart cell built in MATLAB-Simulink. The blocks outlined in green are implemented in an embedded MATLAB function.

Table I: Parameters of the three smart cell simulation study.

\begin{tabular}{ccc}
\hline Description & Parameter & Value \\
\hline \hline \multirow{3}{*}{ Battery Cell Capacities } & $C_{1}$ & $0.75 \mathrm{Ah}$ \\
\cline { 2 - 3 } & $C_{2}$ & $1.20 \mathrm{Ah}$ \\
\cline { 2 - 3 } & $C_{3}$ & $3.00 \mathrm{Ah}$ \\
\hline \multirow{3}{*}{ Smart Cell Parameters } & $L_{s c}$ & $100 \mu \mathrm{H}$ \\
\cline { 2 - 3 } & $f_{s}$ & $50 \mathrm{kHz}$ \\
\cline { 2 - 3 } & $V_{\text {nom }}$ & $4.19 \mathrm{~V}$ \\
\hline \multirow{2}{*}{ Output } & $C_{\text {out }}$ & $4.7 \mu F$ \\
\cline { 2 - 3 } & $R$ & $23.5 \Omega$ \\
\hline
\end{tabular}

Fig. 5 shows how different characteristics of the system evolve over the length of the simulation. The smart cells initially all turn on at the same time, which produces a very high ac rms ripple current in the inductor, as shown in the inset graphs. At the beginning of the simulation, $I_{\text {Lac-rms }}$ is $34 \mathrm{~mA} \mathrm{rms}$, and the peak-to-peak output voltage, $v_{\text {out-pp }}$, is $64 \mathrm{mV}$. After the optimal switching controller is engaged, the phase shift of each smart cell gradually evolves smoothly into its steady state value. At the end of the simulation, $I_{\text {Lac-rms }}$ and $v_{\text {out }-p p}$ have improved considerably to $10 \mathrm{~mA} \mathrm{rms}$ and 11 $\mathrm{mV}$, respectively.

\section{EXPERIMENTAL RESULTS}

The theory developed in this work was tested in the laboratory with an experimental setup consisting of three smart cells in series, as shown in Fig. 1 using the parameters listed in Table I. The hardware was built around ARM's mbed platform, where each smart cell is implemented with the NUCLEOF401RE board and a custom printed circuit board (PCB) containing the power stage. They were tested using laboratory power supplies. Fig. 6 shows a photograph of the three smart cells built in the laboratory.

The NUCLEO-F401RE board was chosen for its relatively powerful micro-controller, the STM32F401RET6, in order
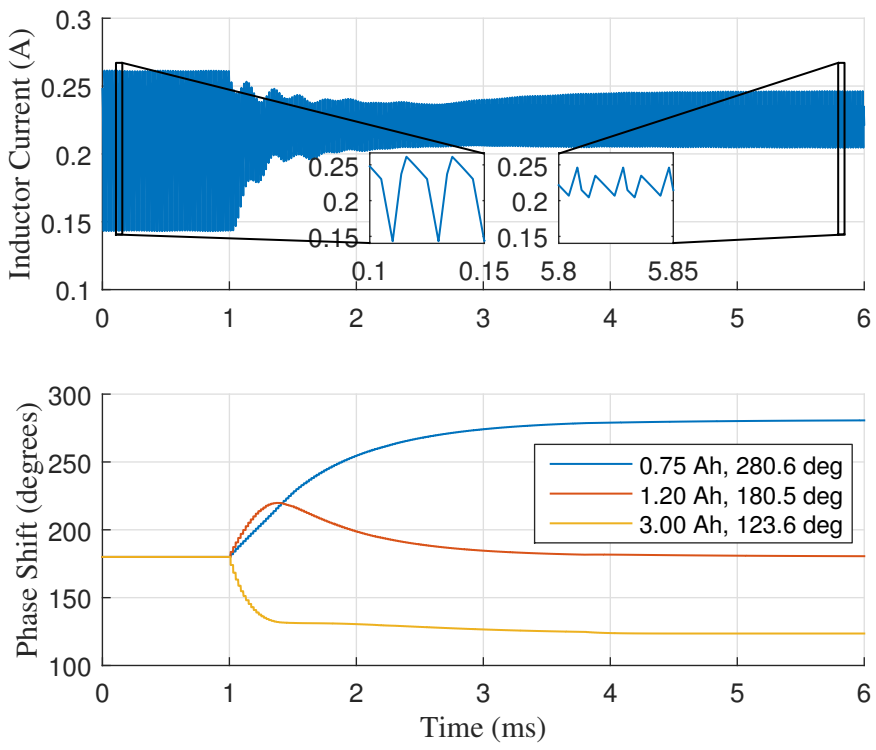

Figure 5: Summary of the simulation results of a three smart cell pack. The smart cell controllers are turned on at $1.0 \mathrm{~ms}$. The ac rms current in the inductor is improved from $34 \mathrm{~mA}$ rms to $10 \mathrm{~mA}$ rms. Although not shown, the peak-to-peak ripple voltage on the capacitor is improved from $64 \mathrm{mV}$ to 11 $\mathrm{mV}$.

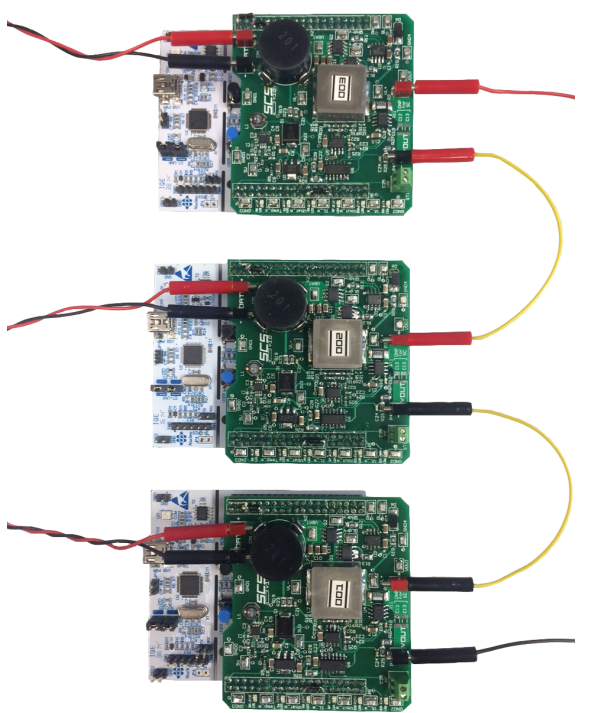

Figure 6: A photograph of the three smart cells tested in the laboratory. The battery cell connections are on the left, and the output is on the right. The smart cells were connected to laboratory power supplies for the tests.

to focus attention on how the smart cell controller can be implemented in hardware. The STM32F401RET6 is based on the ARM 32-bit Cortex-M4 CPU and has a floating point unit.

Fig. 7 shows an oscilloscope screen shot of the three smart cells operating and finding their optimal switching pattern. 


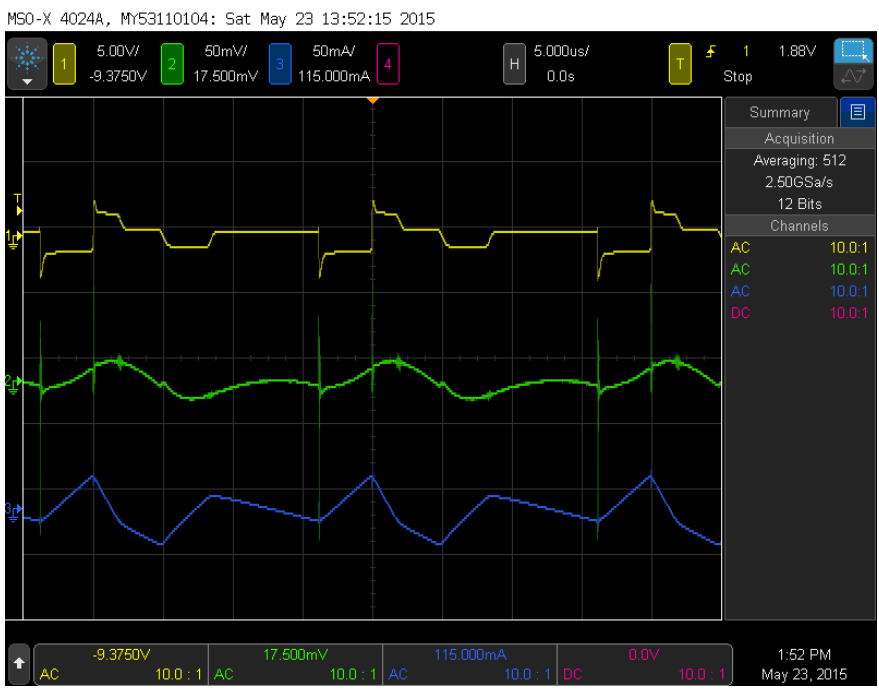

Figure 7: An oscilloscope screen shot of the three smart cells operating at their optimal switching pattern, averaging applied with 512 samples. Channel 1, top yellow, is the voltage across $L_{s c}$ of the first smart cell with a capacity of $C_{1}$. Channel 2, middle green, is the output voltage ripple. Channel 3, bottom blue, is the current in the smart cell inductors.

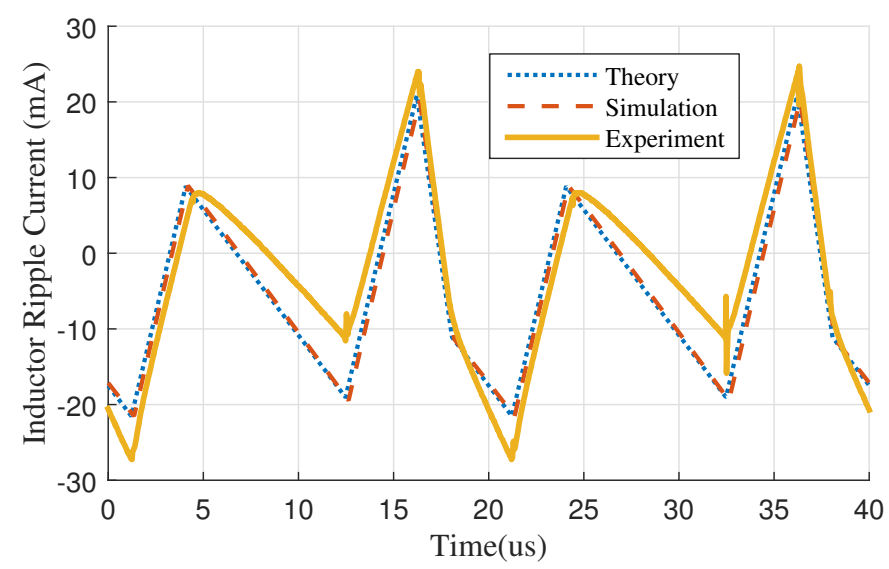

Figure 8: $L_{s c}$ currents from the theoretical analysis, simulation and experiment.

Using the data from the oscilloscope, we can plot the measured $L_{s c}$ current along with the theoretical and simulated $L_{s c}$ currents, and this is shown in Fig. 8. The experimental $L_{s c}$ current matches very well with the theoretical and simulated values, and is well within experimental error when the variation of the inductance of each $L_{s c}$ is taken into account, which is $\pm 20 \%$ for the Vishay Dale IHLP6767GZER101M11 inductor used in these experiments.

The peak-to-peak voltage ripple from the experiments was reduced to $24 \mathrm{mV}$ from $78 \mathrm{mV}$ and the peak-to-peak $L_{s c}$ current ripple was reduced to $54 \mathrm{~mA}$ from $102 \mathrm{~mA}$.

\section{CONCLUSIONS AND FUTURE WORK}

The energy management of series-connected battery cells was addressed with a novel decentralised battery management system based on an MMC topology. Each MMC submodule, or smart cell, operates autonomously within a string to minimize the output ripple voltage and balance the batteries. A smart cell contains a completely decentralized controller, and a small inductor, $L_{s c}$, used to sense transitions in the rest of the string. We developed a theoretical approach to minimising the output voltage ripple by deriving an equation for the ac rms ripple current in the smart cell inductors using Fourier decomposition, taking as inputs the duty cycle and switching phase of each smart cell and minimising this. This result can be applied to MMC converters in general. In order to simplify the approach so that it can be locally applied in a set of autonomous smart cell controllers we also derived a solution that yields near-optimal results by minimising the magnitude of the total sum vector of all smart cell voltages. This is equivalent to only considering the fundamental of the Fourier series of each switching waveform. The theory was demonstrated in simulation and experimental results, where a system of three smart cells were able to find a stable operating point, and the output voltage ripple was reduced from $78 \mathrm{mV}$ to $24 \mathrm{mV}$.

\section{ACKNOWLEDGMENT}

The authors acknowledge the financial support provided by Newtons4th Ltd. (N4L), the Natural Sciences and Engineering Research Council of Canada (NSERC) and Jesus College Oxford. This work also benefited from equipment funded by the John Fell Oxford University Press (OUP) Research Fund and an EPSRC Capital Grant (ref. EP/J021199/1).

\section{REFERENCES}

[1] B. Bose, "Global warming: Energy, environmental pollution, and the impact of power electronics," Industrial Electronics Magazine, IEEE, vol. 4, no. 1, pp. 6-17, March 2010.

[2] — "Power electronics and motor drives recent progress and perspective," Industrial Electronics, IEEE Transactions on, vol. 56, no. 2, pp. 581-588, Feb 2009.

[3] S. Vazquez, S. Lukic, E. Galvan, L. Franquelo, and J. Carrasco, "Energy storage systems for transport and grid applications," Industrial Electronics, IEEE Transactions on, vol. 57, no. 12, pp. 3881-3895, Dec 2010.

[4] S. Wilkinson. The grid-connected energy storage market is set to explode, reaching a total of over $40 \mathrm{gw}$ of installations by 2022 . IHS Technology. [Online]. Available: https://technology.ihs.com/483008/thegrid-connected-energy-storage-market-is-set-to-explode-reaching-atotal-of-over-40-gw-of-installations-by-2022

[5] H. Chen, T. N. Cong, W. Yang, C. Tan, Y. Li, and Y. Ding, "Progress in electrical energy storage system: A critical review," Progress in Natural Science, vol. 19, no. 3, pp. 291 - 312, 2009. [Online]. Available: http://www.sciencedirect.com/science/article/pii/S100200710800381X

[6] D. Andrea, Battery Management Systems for Large Lithium-Ion Battery Packs. Artech House Publishers, October 2010.

[7] J. Kimball, B. Kuhn, and P. Krein, "Increased performance of battery packs by active equalization," in Vehicle Power and Propulsion Conference, 2007. VPPC 2007. IEEE, Sept 2007, pp. 323-327.

[8] J. Zhang, S. Ci, H. Sharif, and M. Alahmad, "Modeling discharge behavior of multicell battery," Energy Conversion, IEEE Transactions on, vol. 25, no. 4, pp. 1133-1141, Dec 2010.

[9] P. Weicker, A Systems Approach to Lithium-Ion Battery Management. Artech House Publishers, October 2013. 
[10] C. Birkl, D. Frost, A. Bizeray, R. Richardson, and D. Howey, "Modular converter system for low-cost off-grid energy storage using second life li-ion batteries," in Global Humanitarian Technology Conference (GHTC), 2014 IEEE, Oct 2014.

[11] M. Rodriguez, Y. Zhang, and D. Maksimovic, "High-frequency pwm buck converters using gan-on-sic hemts," Power Electronics, IEEE Transactions on, vol. 29, no. 5, pp. 2462-2473, May 2014.

[12] D. Reusch, D. Gilham, Y. Su, and F. Lee, "Gallium nitride based 3d integrated non-isolated point of load module," in Applied Power Electronics Conference and Exposition (APEC), 2012 Twenty-Seventh Annual IEEE, Feb 2012, pp. 38-45.

[13] T. McDonald and V. President, "Gan based power technology stimulates revolution in conversion electronics," Electronics in Motion and Conversion, pp. 2-4, 2009.

[14] J. Millan, P. Godignon, X. Perpina, A. Perez-Tomas, and J. Rebollo, "A survey of wide bandgap power semiconductor devices," Power Electronics, IEEE Transactions on, vol. 29, no. 5, pp. 2155-2163, May 2014.

[15] R. A. Leising, M. J. Palazzo, E. S. Takeuchi, and K. J. Takeuchi, “Abuse testing of lithium-ion batteries: Characterization of the overcharge reaction of licoo2/graphite cells," Journal of The Electrochemical Society, vol. 148, no. 8, pp. A838-A844, 2001.

[16] L. Lu, X. Han, J. Li, J. Hua, and M. Ouyang, "A review on the key issues for lithium-ion battery management in electric vehicles," Journal of Power Sources, vol. 226, no. 0, pp. 272 - 288, 2013. [Online]. Available: http://www.sciencedirect.com/science/article/pii/S0378775312016163

[17] A. Lesnicar and R. Marquardt, "An innovative modular multilevel converter topology suitable for a wide power range," in Power Tech Conference Proceedings, 2003 IEEE Bologna, vol. 3, June 2003, pp. 6 pp. Vol.3-.

[18] S. S. Choi and H. S. Lim, "Factors that affect cycle-life and possible degradation mechanisms of a li-ion cell based on licoo2," Journal of Power Sources, vol. 111, no. 1, pp. 130 - 136, 2002. [Online]. Available: http://www.sciencedirect.com/science/article/pii/S0378775302003051 